doi:10.17659/01.2018.0032

Journal of Case Reports 2018;8(2):120-124

\title{
Arterial Bleeding caused by a Dropped Gallstone from a Cholecystoduodenal Fistula
}

Kiyoshi Hotta ${ }^{1}$, Nobuhide Oshitani ${ }^{1}$, Jun-ichi Fujikawa ${ }^{1}$, Hiroko Hata ${ }^{1}$, Takao Sakuma ${ }^{2}$, Masahiro Matsuda ${ }^{1}$, Sanai Noguchi ${ }^{1}$, Yoshimasa Oyama ${ }^{3}$, Yasuhiro Fujiwara ${ }^{4}$

Departments of ${ }^{1}$ Gastroenterology, ${ }^{2}$ Internal Medicine and ${ }^{3}$ Radiodiagnosis; Aizenbashi Hospital, 5-16-15 Nipponbashi, Naniwaku; ${ }^{4}$ Department of Gastroenterology, Graduate School of Medicine, Osaka City University, Osaka-city, Osaka, Japan.

\section{Corresponding Author: \\ Dr. Kiyoshi Hotta \\ Email: k-hotta@tachibana-med.or.jp}

This is an Open Access article distributed under the terms of the Creative Commons Attribution License (creativecommons.org/ licenses/by/3.0).

Received

Accepted

Published

January 5, 2018

April 18, 2018

May 15, 2018

\begin{abstract}
Background: A cholecystoduodenal fistula is often caused by gallstones, but rarely causes gastrointestinal bleeding. Interventional radiology or surgery is used when gastrointestinal bleeding is difficult to treat endoscopically, and interventional radiology has been increasingly performed in recent years. Case Report: We report a rare case of arterial bleeding caused by a dropped gallstone from a cholecystoduodenal fistula, with successful treatment using interventional radiology. Conclusion: Reports of gastrointestinal bleeding from an internal biliary fistula with successful treatment using interventional radiology are very rare. This case illustrates the effectiveness of interventional radiology for a rare case of arterial bleeding caused by a dropped gallstone from a cholecystoduodenal fistula.
\end{abstract}

Keywords: Biliary Fistula, Gallstones, Gastrointestinal Hemorrhage, Intestinal Fistula, Intervention Radiology.

\section{Introduction}

A cholecystoduodenal fistula is often caused by gallstones, but rarely causes gastrointestinal bleeding. Hemostasis for gastrointestinal bleeding is usually performed with endoscopy, but transcatheter arterial embolization or surgery may be required in difficult cases. However, gastrointestinal bleeding from a cholecystoduodenal fistula usually requires urgent surgery.

Here, we report a rare case of arterial bleeding caused by a dropped gallstone from a cholecystoduodenal fistula, with successful treatment using interventional radiology.

\section{Case Report}

A 74-year-old man visited our hospital with sudden malena. His past medical history included gastric cancer (total gastrectomy), myocardial infarction (percutaneous coronary intervention), hypertension, and cholelithiasis. He was taking an antithrombotic drug. His vital signs included blood pressure $87 / 55 \mathrm{mmHg}$, pulse rate 82 beats per minute, and body temperature $36.1{ }^{\circ} \mathrm{C}$. Physical examination showed palpebral conjunctival pallor, soft and non-tender abdomen, and a surgical scar on the mid-abdomen. Laboratory data on admission revealed severe anemia (hemoglobin $6.8 \mathrm{~g} / \mathrm{dL}$ ). Transaminases and biliary tract enzymes were normal [Table 1].

On admission, abdominal computed tomography (CT) [Fig.1] showed duodenal wall thickening and high-density fluid entering the anal side of the intestinal tract; this was thought to be the bleeding source. A gallstone seen on previous CT had disappeared, and a suspected calcified gallstone was seen in the small intestine. Enhanced CT [Fig.2] showed contrast medium leakage into the duodenum. Therefore, we suspected arterial bleeding caused by a falling gallstone from a cholecystoduodenal fistula. 
Table 1: Laboratory examination on admission.

\begin{tabular}{|l|l|l|l|}
\hline \multicolumn{2}{|l|}{ Hematology } & \multicolumn{3}{l|}{ Blood Chemistry } \\
\hline WBC & $5800 / \mathrm{mL}$ & CRP & $1.22 \mathrm{mg} / \mathrm{dL}$ \\
\hline Neutrophils & $84.2 \%$ & Alb & $2.9 \mathrm{~g} / \mathrm{dL}$ \\
\hline Lymphocytes & $8.4 \%$ & T-Bilirubin & $0.3 \mathrm{mg} / \mathrm{dL}$ \\
\hline Monocytes & $4.3 \%$ & Amylase & $114 \mathrm{U} / \mathrm{L}$ \\
\hline Eosinophils & $2.9 \%$ & AST & $35 \mathrm{U} / \mathrm{L}$ \\
\hline Basophils & $0.2 \%$ & ALT & $23 \mathrm{U} / \mathrm{L}$ \\
\hline RBC & $209 \times 10^{4} / \mu \mathrm{L}$ & LDH & $184 \mathrm{U} / \mathrm{L}$ \\
\hline Hemoglobin & $6.8 \mathrm{~g} / \mathrm{dL}$ & BUN & $27 \mathrm{mg} / \mathrm{dL}$ \\
\hline Hematocrit & $21.0 \%$ & Cr & $0.95 \mathrm{mg} / \mathrm{dL}$ \\
\hline Platelet & $15.1 \times 10^{4} / \mu \mathrm{L}$ & & \\
\hline
\end{tabular}

Because of prior total gastrectomy, examination with small intestine balloon endoscopy was required, but was unavailable at our hospital. Due to the emergency condition, referral to another hospital was difficult. Therefore, we attempted transcatheter arterial embolization after obtaining consent from the patient. On angiography [Fig.3], bleeding from the inferior pancreaticoduodenal artery (IPDA), a branch of the superior mesenteric artery (SMA), was found and was successfully stopped with embolization using n-butyl-2cyanoacrylate (NBCA). After hemostasis, rebleeding was not observed and the patient was stable. One day after treatment, a gallstone [Fig.4] was passed by the transanal route. The stone was a $33 \times 30 \mathrm{~mm}$ bilirubin-based gallstone. On hospital day 11, drip-infusion cholangiography-CT [Fig.5] was performed. A cholecystoduodenal fistula was not visualized, and was presumed to have closed spontaneously. The patient did well, and was discharged on hospital day 19.

\section{Discussion}

Internal biliary fistula is a general term for aberrant communication between the biliary tree and adjacent viscera, as first reported by Naunyn in 1892 [1]. A cholecystoduodenal fistula is the most common internal biliary fistula, followed by cholecystocolonic fistula, choledochoduodenal fistula, cholecystogastric fistula, and cholecystocholedochal fistula [2].
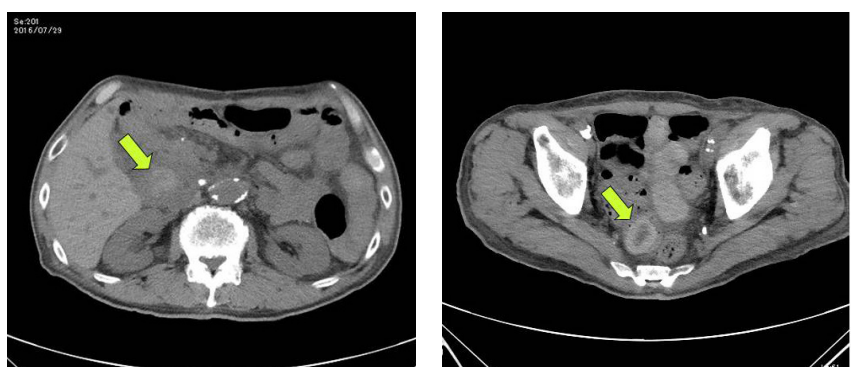

Fig.1(a): Abdominal computed tomography showed a thickened duodenal wall containing high-density fluid. (b): A suspected calcified gallstone was observed in the smallintestine.
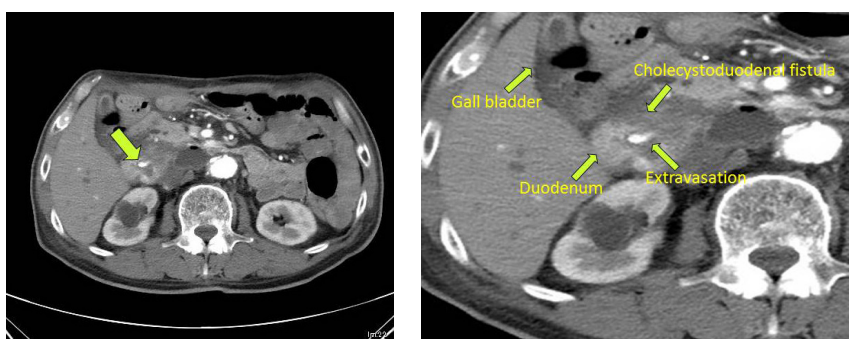

Fig.2(a): Abdominal enhanced computed tomography showed contrast medium leakage into the duodenum. (b): We suspected arterial bleeding caused by a dropped gallstone from a cholecystoduodenal fistula.
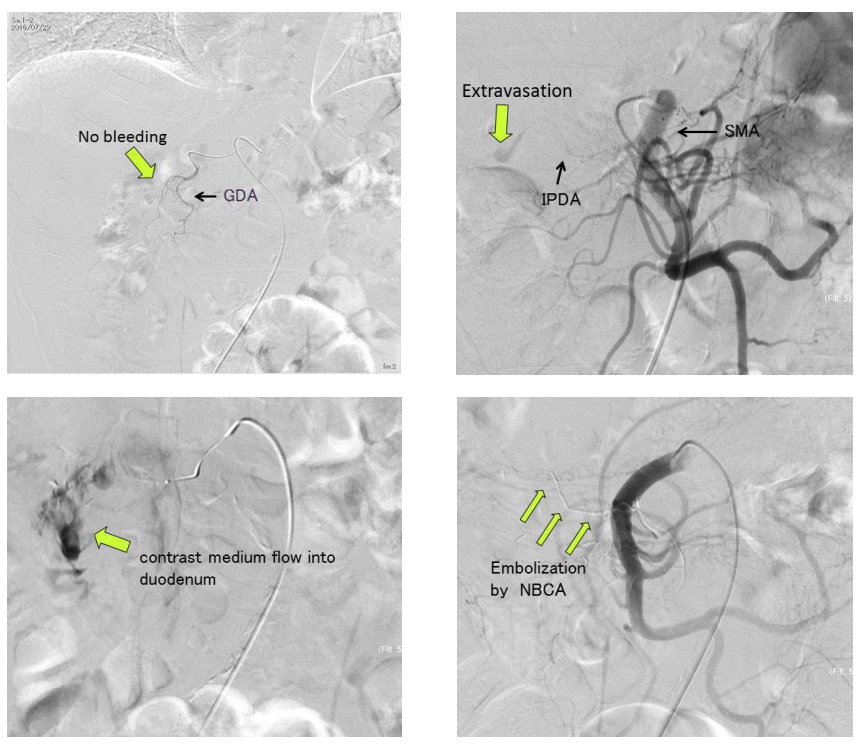

Fig.3(a): On selective gastroduodenal artery (GDA) angiography, extravasation was not visualized. (b): Superior mesenteric artery (SMA) angiography showed a pseudoaneurysm and extravasation from the inferior pancreaticoduodenal artery (IPDA), a branch of the SMA. (c): Leakage of contrast medium into the duodenum was observed. (d): Extravasation was stopped with embolization of the IPDA using n-butyl-2-cyanoacrylate (NBCA). 
Gallstones and cholecystitis are common and account for the development of about $90 \%$ of internal biliary fistulas; other causes include gastroduodenal ulcers and malignancy. Fistulas are thought to develop when chronic gallstone cholecystitis causes adhesion to adjacent viscera; a calculus lodged in the ampulla of the gallbladder then induces a reaction in the wall of the gallbladder, reducing the arterial blood supply and increasing intra-luminal pressure. Finally, necrosis and perforation occur at the site of the ischemia, and a fistula is formed [3].

Symptoms of cholecystoduodenal fistula include fever, jaundice, and abdominal pain due to retrograde infection, but there are few specific symptoms. Rarely, hemobilia occurs in patients with a cholecystoduodenal fistula and gallstone ileus. Abdominal trauma, either accidental or iatrogenic, is the main cause of hemobilia, with gallstones accounting for about $10 \%[4,5]$. However, if minor bleeding is included, gallstones are the main cause of hemobilia [6]. Hemobilia is basically caused by bleeding from an artery into the biliary tract; venous bleeding does not occur, except with portal hypertension or an arterio-venous malformation. In this case, the bleeding site could not be identified with endoscopy. However, we suspected arterial bleeding caused by a falling gallstone from a cholecystoduodenal fistula, because enhanced CT showed contrast medium leakage into the duodenum and a suspected calcified gallstone was seen in the small intestine. During the course of this case, we thought that a cholecystoduodenal fistula had been induced by gallstones and cholecystitis, then later surmised that mechanical damage to a vessel induced by a falling gallstone, antithrombotic therapy, or arteriosclerosis due to aging caused bleeding.

A cholecystoduodenal fistula is usually diagnosed by pneumobilia on abdominal X-ray, abdominal $\mathrm{CT}$ and reverse flow to the biliary tract on upper gastrointestinal contrast radiography. Gallbladder carcinoma can develop in $15 \%$ of
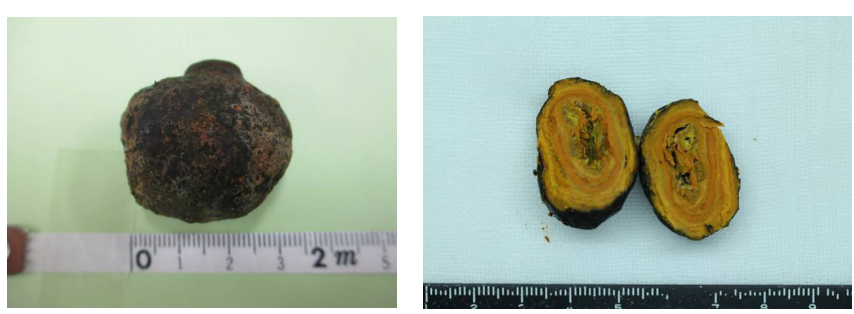

Fig.4: The stone was a $33 \times 30 \mathrm{~mm}$ bilirubin-based gallstone.

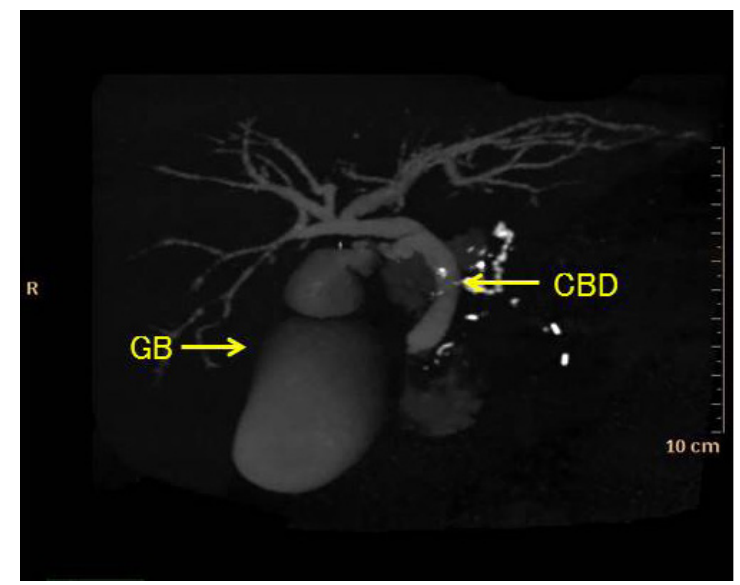

Fig.5: Cholecystoduodenal fistula was not visualized with drip-infusion cholangiography-computed tomography.

cholecystoduodenal fistula cases, and a causal relationship between internal biliary fistula and gallbladder carcinoma has been suggested [7]. Therefore, a cholecystoduodenal fistula should be treated with cholecystectomy and closure of the fistula. Spontaneous closure of a cholecystoduodenal fistula has been reported recently; in the present case, the fistula closed spontaneously within 11 days after treatment with interventional radiology, thus avoiding biliary surgery. There are many reports of spontaneous closure of an internal biliary fistula when there is no remaining stone in the biliary tract and the internal pressure in the biliary tract is decreased [8]. Thus, depending on patient age and general condition, an internal biliary fistula may be observed without surgery.

Hemobilia from a cholecystoduodenal fistula usually requires surgery (ligature hemostasis, cholecystectomy and closure of the 
Table 2: Case reports of gastrointestinal bleeding from an internal biliary fistula successfully treated with interventional radiology.

\begin{tabular}{|l|l|l|l|l|l|l|}
\hline Year / Author & Age / Gender & Type of fistula & Symptoms & Bleeding vessel & $\begin{array}{l}\text { Method of } \\
\text { embolization }\end{array}$ & $\begin{array}{l}\text { Antithrombotic } \\
\text { drug }\end{array}$ \\
\hline $2009 /$ Mashim $^{9}$ & $87 / \mathrm{F}$ & CDF & $\begin{array}{l}\text { Hematemesis, } \\
\text { gall stone }\end{array}$ & GDA & N.D. & + \\
\hline $2014 /$ Oono $^{10}$ & $92 / \mathrm{F}$ & CDF & $\begin{array}{l}\text { Hematemesis, } \\
\text { gall stone, } \\
\text { hemorrhagic shock }\end{array}$ & CA & coil & + \\
\hline $2017 /$ this case & $74 / \mathrm{M}$ & CDF & Malena, gall stone & IPDA & NBCA & + \\
\hline
\end{tabular}

CDF: cholecystoduodenal fistula; GDA: gastroduodenal artery; CA: cystic artery; IPDA: inferior pancreaticoduodenal artery; ND: No description; NBCA: n-butyl-2-cyanoacrylate.

fistula). However, in an elderly patient, circulatory instability is a concern, and conservative treatment with less invasive interventional radiology for hemostasis can be performed. Interventional radiology or surgery is used when gastrointestinal bleeding is difficult to treat endoscopically, and interventional radiology has been increasingly performed in recent years. In this case, angiography showed extravasation from the IPDA and bleeding was successfully stopped after embolization using NBCA. Post-hemostasis complications such as rebleeding and intestinal necrosis were not observed and a good prognosis was obtained. Treatment with interventional radiology is safe and effective as a less-invasive alternative to surgery, but requires the ability to reach the target vessel with a catheter. Thus, depending on the status of the blood vessel and location of the lesion, there are cases in which interventional radiological treatment is difficult. Furthermore, it is important to selectively perform embolization on the bleeding vessel to prevent intestinal necrosis caused by excessive embolization.

A PubMed search did not find a report of a patient with gastrointestinal bleeding from an internal biliary fistula successfully treated with interventional radiology. However, a search of Japanese literature found three reports, including the present case [Table 2]. All patients were elderly, taking antithrombotic drugs, and had cholecystoduodenal fistulas with gallstones. The bleeding vessel was the gastroduodenal artery (GDA), the cystic artery (CA), or the IPDA, and the embolization method was coil and NBCA with an unknown method in one case.

\section{Conclusion}

Formation of a cholecystoduodenal fistula by compression and stimulation from a gallstone is sometimes experienced, but a cholecystoduodenal fistula is a rare cause of gastrointestinal bleeding. This case was significant, as arterial bleeding caused by a falling gallstone from a cholecystoduodenal fistula was successfully treated using interventional radiology.

Contributors: KH: manuscript writing, literature search, patient management; NO, JF, HH, TS, MM, SN: participation in clinical discussion of the patient, critical inputs in manuscript; YO: contribution to intervention radiology and manuscript editing; YF: supervision to data collection and references. KH will act as guarantor. All authors approved the final version of this manuscript.

Funding: None; Competing interests: None stated.

\section{References}

1. Naunyn B. Klinik der cholelithiasis. F.C.W. Vogel, Leipzig. 1892:139-141.

2. Ozdemir A, Atli MY, Coskun T, Ozenc A, Hersek E. Biliary enteric fistulas. Int Surg. 1997;82:280-283.

3. Glenn F, Reed C, Grafe WR. Biliary enteric fistula. Surg Gynecol Obstet. 1981;153:527-531.

4. Sandblom P. Hemobilia. Surg Clin North Am. 1973;53:1191-1210.

5. Czerniak A, Thompson JN, Hemingway AP, Soreide O, Benjamin IS, Alison DJ, et al. Hemobilia. A disease in evolution. Arch Surg.1988;123:718-721.

6. Sandblom P, Mirkovitch V. Minor hemobilia: clinical significance and pathophysiological background. Ann Surg. 1979;190:254-264. 
7. Berliner SD, Bruson LC. One stage repair for cholecystoduodenal fistula and gallstone ileus. Arch Surg. 1965;90:313-316

8. Raf L, Spangen L. Gallstone ileus. Acta Chir Scand. 1971;137:665-675.

9. Mashimo M, Hara J, Hotta K, Kawachiya T, Tamamori $\mathrm{Y}$, Teraoka $\mathrm{H}$. Proceedings of the $77^{\text {th }}$ Annual Meeting of the Japan Gastroenterological Endoscopy Society Conference. 2009 May 21-23;Nagoya City, Japan.

10. Oono $K$, Hiraaki E, Yauti $M$, Nagata $H$, Yamaji $O$, Uraushihara K. A case of cholecystoduodenal fistula presenting with gastrointestinal bleeding. Tama Symposium Journal of Gastroenterology. 2015;29:1723. 